\title{
A NUMERICAL CALCULATION OF THE GENERAL CIRCULATION OF THE ATMOSPHERE
}

$\mathrm{M}$ UCH work has been done in recent years in the calculation of changes in the wind and temperature of the atmosphere from hydrodynamic and thermodynamic theory. The equations are nonlinear partial differential ones and, moreover, the atmosphere is a turbulent fluid of variable viscosity, subject to heating and cooling by radiation and changes of state of water and retarded at the ground by surface friction. It is now well realized that only by applying dynamical theory to the atmosphere can serious progress be made, and the method is the classical scientific one of studying simplified model atmospheres and comparing the results with observations.

Hitherto, the models used have not allowed for friction or for heating and cooling by radiation. In such models, temperature changes adiabatically only in association with pressure. Now, however, in a paper in the April number of the Quarterly Journal of the Royal Meteorological Society, which has since been awarded the first Napier Shaw Memorial Prize, N. A. Phillips, of the Institute of Advanced Study, Princeton, describes an experiment in the numerical calculation of atmospheric changes allowing for friction and non-adiabatic heating. Phillips starts with an atmosphere at rest relative to the Earth and at a uniform temperature, and calculates developments. The calculation is based on approximate equations of motion and thermodynamic energychange. The upper atmosphere is represented by the $250-\mathrm{mb}$. level and the lower by the $750-\mathrm{mb}$. one. The surface of the Earth and its associated friction and zero vertical velocity is everywhere at $1,000 \mathrm{mb}$. The divergence at the $750-\mathrm{mb}$. level is necessarily equal and opposite in sign to that at $250 \mathrm{mb}$. Important assumptions are : hydrostatic equilibrium in the vertical ; geostrophic wind except in one term of the equations; constancy of the Coriolis parameter and its derivative with respect to latitude; and constancy with height of the stability parameter $\left(\theta_{1}-\theta_{3}\right) / \theta_{2}$, where $\theta_{1}, \theta_{2}, \theta_{3}$ are potential temperatures at 250,500 and $750 \mathrm{mb}$.

The atmosphere is supposed to be heated by radiation from the equator to latitude $45^{\circ}$ and cooled from there to the pole at a rate independent of height but proportional to distance from latitude $45^{\circ}$. Friction at the ground and lateral and vertical diffusion are allowed for with reasonable assumptions. Empirical mean values are used for the various constants. The equations of motion and energy are reduced to finite difference form and solved on an electronic computer for values at a grid of points at intervals of $625 \mathrm{~km}$. latitudinally and $375 \mathrm{~km}$, longitudinally.

Calculations were made over a rectangular area of width $6,000 \mathrm{~km}$. from west to east and depth 10,000 $\mathrm{km}$. from south to north. The boundary conditions were : $(a)$ identity of values of all elements at a given latitude on the east and west boundaries ; $(b)$ zero normal geostrophic wind and zero disturbed geostrophic vorticity on the south and north boundaries.

Starting from the state of rest and uniform temperature and with the restraint that the flow was independent of longitude, the flow and temperature patterns were first computed step by step for a period of 130 days, by the end of which time there were a nearly uniform fall of temperature from equator to pole, broad westerly winds with the reasonable maximum of $36.3 \mathrm{~m} . / \mathrm{sec}$. in middle latitudes at $250 \mathrm{mb}$, a weak easterly wind (order $1 \mathrm{~m} . / \mathrm{sec}$.) at the surface and a minute southerly wind at $250 \mathrm{mb}$. On this circulation there was then superimposed a small random flow initially independent of height but varying borizontally in a random manner, and the development again computed. This culminated in the formation of a large central depression moving eastward at about $1,800 \mathrm{~km}$./day and having features, such as isobars like those at fronts, corresponding to real depressions and with a marked westerly jet-stream and a mean poleward flux of energy.

Considering the assumptions made, the result must be regarded as a remarkably close approximation to reality, and the paper is one to be read by all meteorologists and mathematicians interested in that most difficult problem of hydrodynamics, the circulation of the atmosphere.

\section{INDUSTRIAL APPLICATIONS OF THE ELECTRON MICROSCOPE}

A CONFERENCE on "Industrial Applications of the Electron Microscope", organized by l'Association des Ingénieurs sortis de l'École de Liège, was held in Liège during May 2-5. Representatives of fourteen countries were present. The main part of the conference was devoted to the study of metals and one session to chemical problems. Throughout the conference it was noteworthy that, although applications of the electron microscope were described in all papers, in few was the microscope given undue prominence. It appears that the electron microscope is now being used as just one more piece of apparatus in the laboratory. The results obtained are valuable and, in many cases, not obtainable in any other way; but the time has passed when problems are being sought specifically to find a use for the electron microscope.

Dr. L. Habraken (Centre National de Recherches Métallurgiques, Liège) welcomed the visitors on behalf of the Association and of the C.N.R.M. and introduced the first speaker, Dr. J. B. Le Poole (Technisch Hoogeschool, Delft), who traced the history and probable future of electron microscopes. Early electron microscopes were merely optical analogues of light microscopes; the resolution obtainable to-day had only been achieved when methods of lens correction specifically applicable to electrons and not to light had been applied. New methods in the optics of charged particles, in particular, devices using velocity 